\title{
Fuzzy Versus Neuro-fuzzy Identification of Active Front Steering of a Vehicle on Slippery Roads
}

\author{
B. Aalizadeh ${ }^{1}$ and A. Asnafi ${ }^{2}$,* \\ ${ }^{1}$ Department of Mechanical Engineering, Shahid Chamran University of Ahvaz, \\ Ahvaz, Iran \\ ${ }^{2}$ Hydro-Aeronautical Research Center, Shiraz University, Shiraz, 71348-13668, Iran. \\ *E-mail: asnafi@shirazu.ac.ir \\ Phone: +98-71-336133771, Fax: +98-71-32270296
}

\begin{abstract}
Due to the high rate changes in the handling of cars, the use of an auxiliary identification process to design efficient controllers is of importance. Many identification algorithms have been proposed in the literature, which generally performs well under normal situations, but does not show acceptable performance in uncertain conditions. In this article, due to the nature of the neuro-fuzzy networks in identifying and predicting uncertain conditions, an adaptive neuro-fuzzy identification algorithm is proposed to steer vehicles at the uncertain slippery condition of roads. A set of data for three well-known manoeuvres of vehicle dynamics at conventional conditions was collected to train the algorithm using adaptive neuro-fuzzy inference system of MATLAB. Using back propagation of error as the learning algorithm, the parameters of the algorithm were modified regarding uncertain conditions. Making an analogy, the performance of the proposed identification scheme was compared to the untrained fuzzy one. In regular situations, the results were almost identical, but in uncertain ones such as slippery roads, the performance of the proposed neuro-fuzzy algorithm was much better.
\end{abstract}

Keywords: Fuzzy logic system; neuro fuzzy identification; artificial neural networks; vehicle handling; front steering control.

\section{INTRODUCTION}

One of the most important factors in controlling the stability and handling of vehicles is its steering system. The stability and handling of a vehicle may be affected by several uncertain conditions such as vehicle speed, road coefficient of friction and total weight of vehicle [1]. One of the indicators of stability assessment in vehicles is the behaviour of their yaw rates in actual manoeuvres. Several methods have been proposed to control the yaw rate of a vehicle, including the use of direct yaw moment control, four-wheel steering system, active differential braking system, and active front steering system (AFS) [2]. The latter improves the vehicle's manoeuvrability, stability and path tracking by compensating an additional angle to the one that the driver has already applied [3].

In recent decades, some nonlinear control techniques to control the complex steering system of vehicles have been proposed. Due to the high cost of practical tests, the first step is to model the behaviour of the steering system with numerical simulations. There are two main problems in these modelling and simulations; one is to decrease the error between the response of a real vehicle and its corresponding model and, the other is to overcome the uncertain conditions that a vehicle might face. Many of the proposed 
models are able to meet the first demands, but generally, do not perform well under uncertain conditions (for example change of the coefficient of friction of the roads). Logically and theoretically, it is expected that an adaptive set including identification and control can satisfy these requirements simultaneously. We have two approaches to resolving this issue, using an accurate controller with all the details that are costly to implement, or using a cheap and adaptive identification algorithm that can provide the same performance alongside a simpler controller.

Several studies have been conducted to simulate the steering system of vehicles using an identification method or a simple or complex mathematical model. Kazemi et al. [4] assumed a 7-degree of freedom model for the steering system neglecting the roll and pitch motions. Although, the simple model presented in the above article provides the purpose of the paper, but with the change in the condition of the problem, the performance of the model is not guaranteed. Messoussi et al. used a 10-degree of freedom model and a nonlinear controller (Takagi Sugeno fuzzy model) to improve both the stability and handling of a 4-wheel drive (4WD) steering system [5]. Jian Ma et al. [6] designed a fuzzy logic controller for an 8-degree of freedom car that uses an active front steering system (AFS) for manipulation. Of course, this model did not take into account the effect of uncertain road changes.

Using neural networks that utilised backpropagation of error as a learning method, Wu et al. [7] arranged a process to identify a vehicle model from its dynamics. The model used a four-wheel steering system and a fuzzy controller optimised by a genetic algorithm. They designed the controller for a specific condition whereas the car is always under predictable conditions. Some works have been reported in the literature to model the yaw rate response of vehicles in different conditions. Canale et al. [8] modelled a vehicle and then proposed a robust controller to manipulate the yaw rate of a vehicle in some certain situations. No identification and adaptation were employed; thus, good performance was achieved in only definite ranges of uncertainties. Wang et al. [9] exploited a three-degree of freedom model to identify vehicle handling response using a neural network method under uncertain disturbances. It is to be noted that the 3-DOF model used in their work to train the identification algorithm was not an almost precise model.

Zhang and Wang [10] proposed a controller-gain tuning approach integrating active front steering system and direct yaw moment to enhance vehicle handling under velocity change uncertainties while the road coefficient of friction was not considered as a source of uncertainty. Aalizadeh and Asnafi $[11,12]$ studied the stability and handling of a specific vehicle in uncertain road condition using a combination of artificial neural networks and bio-inspired optimisation algorithms. Aalizadeh [13] investigated the effects of uncertain road conditions on vehicle handling and proposed a neuro-fuzzy controller to enhance system performance. The latter work used the general logic rules behind the proposed identification algorithm in this study to design an efficient controller rather than to develop a precise identification algorithm.

To meet both the requirements, i.e. using a more realistic model and to consider uncertain conditions, an adaptive neuro-fuzzy identification algorithm, as a conjecture, is proposed. It is to be noted that the neuro-fuzzy systems use the advantages of both decision making of fuzzy systems and adaptive nature of neural networks simultaneously. The suggested algorithm is able to adapt its parameters according to unpredictable road conditions within a short period, as it happens in real cases. 


\section{MATERIALS AND METHODS}

\section{Vehicle Model Description}

In this research, an almost complete multi-body model of a vehicle in CARSIM which is a simulation toolbox of MATLAB SIMULINK is used. The main advantage of this model is accurate modelling of the vehicle using a largely enough number of degrees of freedom. The parameters of the vehicle used in this study are shown in Table 1 . In this table, $M, V$, and $I$ are the nominal mass, speed, and yaw mass moment of inertia. $R_{w}$ is the nominal radius of whthe eel, $I_{w}$ is the mass moment of inertia of wheel, $H$ is the height of the center of gravity of sprung mass, $l_{f}$ and $l_{r}$ are the distances between fronthe t/rear wheel and the center of mass point, and $l_{w}$ is the width between right and left wheels.

Table. 1. The parameters of the vehicle used in this study.

\begin{tabular}{llllll}
\hline$M$ & $1530(\mathrm{~kg})$ & $l_{f}$ & $1.14(\mathrm{~m})$ & $R_{w}$ & $0.31(\mathrm{~m})$ \\
$I$ & $4607.5\left(\mathrm{~kg} \cdot \mathrm{m}^{2}\right)$ & $l_{r}$ & $1.64(\mathrm{~m})$ & $I_{w}$ & $0.9\left(\mathrm{~kg} \cdot \mathrm{m}^{2}\right)$ \\
$V$ & $72(\mathrm{~km} / \mathrm{h})$ & $l_{w}$ & $1.55(\mathrm{~m})$ & $H$ & $0.52(\mathrm{~m})$ \\
\hline
\end{tabular}

\section{Identification of Model}

To overcome the second problem i.e. reaching satisfactory performance at uncertain conditions, an identification process which is able to modify and adapt the algorithm parameters during manoeuvres is required. Here, a fuzzy logic system which takes advantage of the neural network to adapt its parameter according to unpredictable changes is employed (see following sections for more details). In this study, the backpropagation of error $[11,12]$, as a training algorithm, is selected. More specifically, the input and output data of the system under study are collected for three well-known manoeuvres i.e. lane change, j-turn, and fish-hook in a dry road condition. These data are then transferred to ANFIS (adaptive neuro-fuzzy inference system) toolbox of MATLAB software as a set of training data. Since this fuzzy system can only predict the behaviors properly for cases that has been trained for, it cannot be used alone; thus, a neural network is combined with this fuzzy system to enrich the identification logic so that the adaptation of parameters for other uncertain conditions can also be achieved. Briefly, it is a fuzzy system of Sugeno type which modifies output parameters of its rules using backpropagation of error as the learning algorithm. A schematic of the identification algorithm is shown in Figure 1. In this figure, signal variables st, a, and v are steering angle, lateral acceleration, and lateral velocity of the vehicle respectively. Steering angle (st) is an input for both the identification system and the vehicle. Signals y (vehicle yaw rate), $\mathrm{a}$, and $\mathrm{v}$ are the vehicle output signal while $\mathrm{a}$, and $\mathrm{v}$ enter to identification system as input signals also. The error is now the difference between the actual yaw rate of the vehicle $(y)$ and the one computed by the identification algorithm $(\bar{y})$. 


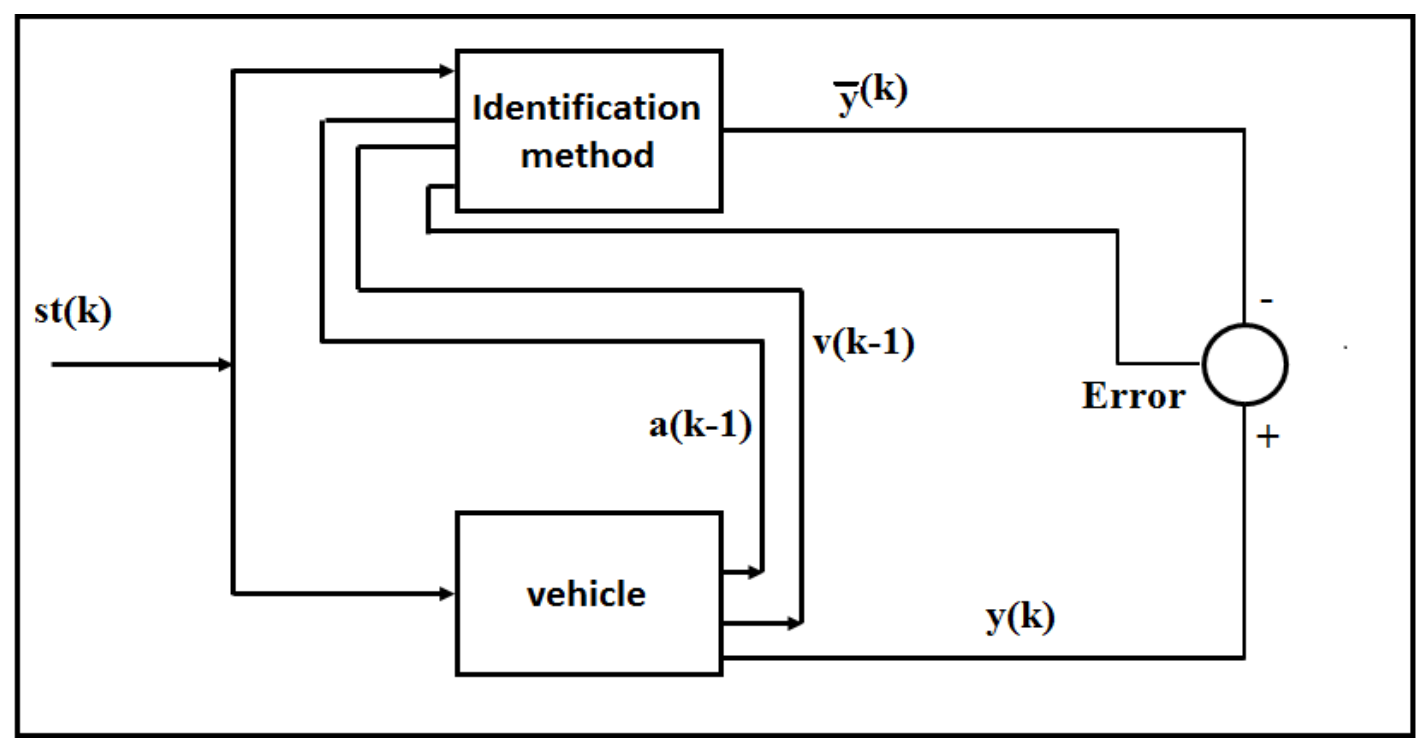

Figure 1. A schematic diagram of the proposed identification system.

\section{The Structure of Fuzzy Logic System}

Fuzzy logic was first proposed by Zadeh in the 1960's, as an inspiration from the brain's thinking and decision-making process [14]. This logic has been widely used in many applied sciences. As an example, see references [15-18] that used this logic in the control and identification fields. There are many fuzzy interface methods in the literature; among them, The Mamdani's [15] and Sugeno [16] interface methods are most well-known. In this study, a fuzzy logic system interface of Sugeno type that was first proposed by Takagi and Sugeno is used [16]. The structure of the fuzzy identification algorithm of Sugeno type (also known as TSK) is shown schematically in Figure 2. (see also [17] for more details).

Generally, consider $\mathrm{x}_{1}, \mathrm{x}_{2}$, and $\mathrm{x}_{3}$ are the input signals of fuzzy systems. In our model, they are the steering angle, lateral acceleration and lateral velocity of the vehicle respectively. In the first layer, using Gaussian membership functions, the fuzzification process is done so that the output signals of the first layer become:

$$
\begin{aligned}
& \mathrm{O}_{\mathrm{i}}^{(1)}=\mathrm{A}_{\mathrm{i}}\left(\mathrm{x}_{1}\right), \mathrm{i}=1, \ldots, 3 \\
& \mathrm{O}_{\mathrm{j}}^{(1)}=\mathrm{B}_{\mathrm{j}}\left(\mathrm{x}_{2}\right), \mathrm{j}=1, \ldots, 3 \\
& \mathrm{O}_{\mathrm{k}}^{(1)}=\mathrm{C}_{\mathrm{k}}\left(\mathrm{x}_{3}\right), \mathrm{k}=1, \ldots, 3
\end{aligned}
$$

where $A_{i}\left(\mathrm{x}_{1}\right), B j\left(\mathrm{x}_{2}\right)$, and $C_{k}\left(\mathrm{x}_{3}\right)$ are the membership functions of the input functions respectively, and $O^{(1)}$ 's represent the output signals of the first layer. At the second layer, using all multiplicative combinations of the first layer output signals, a set of weight signals for the TSK fuzzy rules is prepared so that the output signals of the second layer become: 


$$
\begin{aligned}
& \mathrm{O}_{1}^{(2)}=\mathrm{w}_{1}=\mathrm{A}_{1}\left(\mathrm{x}_{1}\right) \times \mathrm{B}_{1}\left(\mathrm{x}_{2}\right) \times \mathrm{C}_{1}\left(\mathrm{x}_{3}\right) \\
& \mathrm{O}_{2}^{(2)}=\mathrm{w}_{2}=\mathrm{A}_{1}\left(\mathrm{x}_{1}\right) \times \mathrm{B}_{1}\left(\mathrm{x}_{2}\right) \times \mathrm{C}_{2}\left(\mathrm{x}_{3}\right) \\
& \cdot \\
& \cdot \\
& \mathrm{O}_{27}^{(2)}=\mathrm{w}_{27}=\mathrm{A}_{3}\left(\mathrm{x}_{1}\right) \times \mathrm{B}_{3}\left(\mathrm{x}_{2}\right) \times \mathrm{C}_{3}\left(\mathrm{x}_{3}\right)
\end{aligned}
$$

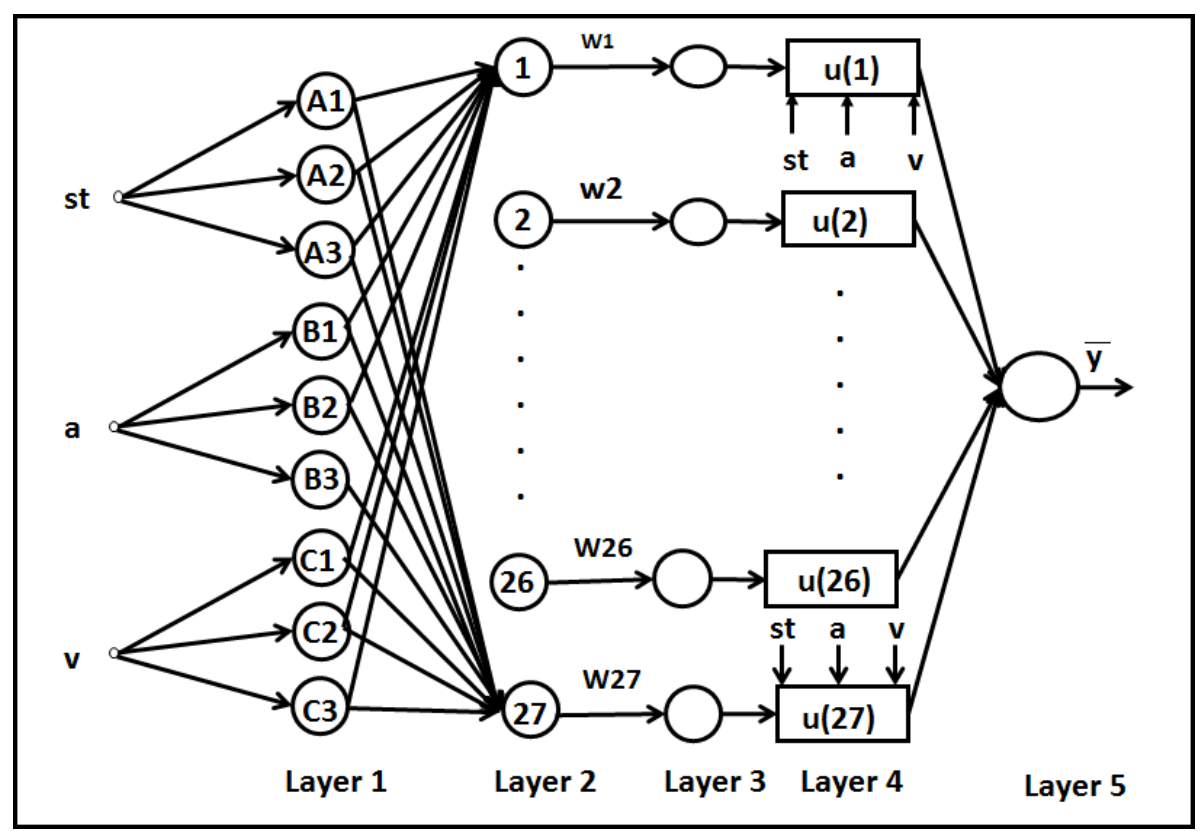

Figure 2. The structure of the fuzzy system interface of Sugeno type.

In the third layer, the weight signals of each rule are normalised as follow:

$$
O_{i}^{(3)}=\bar{w}_{i}=\frac{\mathrm{w}_{i}}{\sum \mathrm{w}_{i}} i=1, \ldots, 27
$$

Making a simple analogy, for a three-input fuzzy system, the rules of TSK (Sugeno type) fuzzy model are (see also [14]):

$$
\mathrm{z}_{\mathrm{i}}=\mathrm{p}_{\mathrm{i}} \mathrm{x}_{1}+\mathrm{q}_{\mathrm{i}} \mathrm{x}_{2}+\mathrm{r}_{\mathrm{i}} \mathrm{x}_{3}+\mathrm{s}_{\mathrm{i}} \mathrm{i}=1, \ldots, 27
$$

where $\mathrm{x}_{1}-\mathrm{x}_{3}$ are the input signals of the fuzzy system and parameters $\mathrm{p}_{\mathrm{i}}, \mathrm{q}_{\mathrm{i}}, \mathrm{r}_{\mathrm{i}}$, and $s_{i}$ are the corresponding constant values for the $\mathrm{i}^{\text {th }}$ rule. The output signals of each rule are determined in the fourth layer as:

$$
\mathrm{O}_{\mathrm{i}}^{(4)}=\overline{\mathrm{w}}_{\mathrm{i}} \mathrm{z}_{\mathrm{i}}=\overline{\mathrm{w}}_{\mathrm{i}}\left(\mathrm{p}_{\mathrm{i}} \mathrm{x}_{1}+\mathrm{q}_{\mathrm{i}} \mathrm{x}_{2}+\mathrm{r}_{\mathrm{i}} \mathrm{x}_{3}+\mathrm{s}_{\mathrm{i}}\right), \mathrm{i}=1, \ldots, 27
$$

Finally, the entire output of the fuzzy system is determined in the fifth layer as: 


$$
\bar{y}=O_{i}^{(5)}=\sum_{i=1}^{27} \bar{w}_{i} z_{i}=\frac{\sum \bar{w}_{i} z_{i}}{\sum W_{i}}
$$

\section{Training Algorithm for the Neuro-Fuzzy System}

The goal of this study is to design a fuzzy identification algorithm that can modify its parameters using a learning algorithm. As previously indicated, among the numerous algorithms, artificial neural networks are the best choice in this regard [18]. Neural networks appeared in the 1940's when first mathematical models of neurons were presented by McCulloch and Pitts [19] and then by Hebb's organisation [20]. These networks are designed to learn and do trained tasks similar to what a biological brain does. In an artificial neural network, the layers of neurons and some connections (synapse and post-synapse) between them are defined. Firstly, a set of known input/output data is used to train the network. After that, the network is expected to find the appropriate response for a new input relative to its information background; exactly as it does in the process of experiential learning. The efficiency and accuracy of neural networks has been discussed in several studies in the literature (see for example [11, 12]).

Usually, the mechanism for finding the best answer is performed by minimising a cost function that correlates the identified model response and the actual system. Here, the cost function is defined as:

$$
\mathrm{E}=\frac{1}{2} \sum_{\mathrm{n}=1}^{\mathrm{L}} \mathrm{e}^{2}(\mathrm{n})
$$

where (see also Fig. 1):

$$
\mathrm{e}(\mathrm{n})=\mathrm{y}-\overline{\mathrm{y}}(\mathrm{n})
$$

In this research, the backpropagation of error [21] is used to minimise the cost function (Eq. 9). This algorithm has been widely employed to train artificial neural networks in the literature (see for example [21]). The algorithm was first introduced by Werbos [22] and then used as a training algorithm in artificial neural networks [23].

Using delta learning rule $[24,25]$ for minimising the error $E$, the gradient of the TSK rules (Eq. 6) must be in the opposite direction of the gradient of the error $E$ i.e.

$$
\Delta \mathrm{z}_{\mathrm{i}} \propto-\frac{\partial \mathrm{E}}{\partial \mathrm{z}_{\mathrm{i}}} \stackrel{\text { Chain rule }}{=}-\frac{\partial \mathrm{E}}{\partial \mathrm{e}(\mathrm{n})} \frac{\partial \mathrm{e}(\mathrm{n})}{\partial \overline{\mathrm{y}}(\mathrm{n})} \frac{\partial \overline{\mathrm{y}}}{\partial \mathrm{u}} \frac{\partial \mathrm{u}}{\partial \mathrm{z}_{\mathrm{i}}}
$$

Finally, the following relation for the weight modification procedure can be obtained:

$$
\Delta \mathrm{z}_{\mathrm{i}}=\eta \mathrm{e}(\mathrm{n}) \frac{\partial \mathrm{u}}{\partial \mathrm{z}_{\mathrm{i}}}
$$

where $\eta$ is known as the coefficient of training. In the training simulation, both the speed of the process and the convergence factors must be satisfied; so, the value of 0.9 is chosen for parameter $\eta$. The practical update rule becomes: 


$$
z_{i}(n+1)=z_{i}(n)+\eta e(n) \frac{\partial u}{\partial z_{i}}
$$

The identification process was performed as follows: Firstly, a set of training data was obtained from the response of a reference vehicle in CARSIM under three wellknown manoeuvres, i.e. the lane change, the fish-hook, and the J-turn on a dry road with a coefficient of friction equal to one. Secondly, the given fuzzy logic system was trained using the adaptive neuro-fuzzy inference system (ANFIS) toolbox of MATLAB. Finally, the parameters of the TSK rule in Eq. (8) were considered to be updated similar to that was presented in Eq. (13) as:

$$
\begin{aligned}
& p_{i}(n+1)=p_{i}(n)+\eta e(n) \frac{\partial u}{\partial p_{i}} \\
& q_{i}(n+1)=q_{i}(n)+\eta e(n) \frac{\partial u}{\partial q_{i}} \\
& r_{i}(n+1)=r_{i}(n)+\eta e(n) \frac{\partial u}{\partial r_{i}} \\
& s_{i}(n+1)=s_{i}(n)+\eta e(n) \frac{\partial u}{\partial s_{i}}
\end{aligned}
$$

\section{RESULTS AND DISCUSSION}

In this section, the response of the pointed vehicle under the action of the designed neurofuzzy identification algorithm on dry and slippery roads is investigated. Three standard classical manoeuvres, i.e. the J-turn, the fishhook, and the lane change are selected.

In lane change manoeuvre, the car simply changes its lane while in J-turn one, it makes a simple turn with a constant radius of rotation. In fish-hook manoeuvre, which is a combination of both, after changing the lane to one direction, the turn is done in the other direction, for example, changing the lane to the right one and then turning to the left with a constant radius of rotation. Figure 3 shows the evolution of the steering angle of the front wheel in these manoeuvres.

\section{Vehicle Response in the Prescribed Manoeuvres}

The coefficient of friction as a measure for uncertain conditions was selected. In our simulations, this value for dry and slippery roads was considered to be 1 and 0.4 , respectively. In what follows, the desired evolution of the yaw rate of the vehicle during these manoeuvres is drawn and compared to those are obtained under the action of fuzzy and neuro-fuzzy identification algorithm. In Figure 4, the desired (real) response of the vehicle and the ones under the action of fuzzy and neuro-fuzzy identification for the lane change manoeuvre at different road conditions are drawn. Figures 5 and 6 also present the same results for J-turn and fish-hook manoeuvres respectively. 


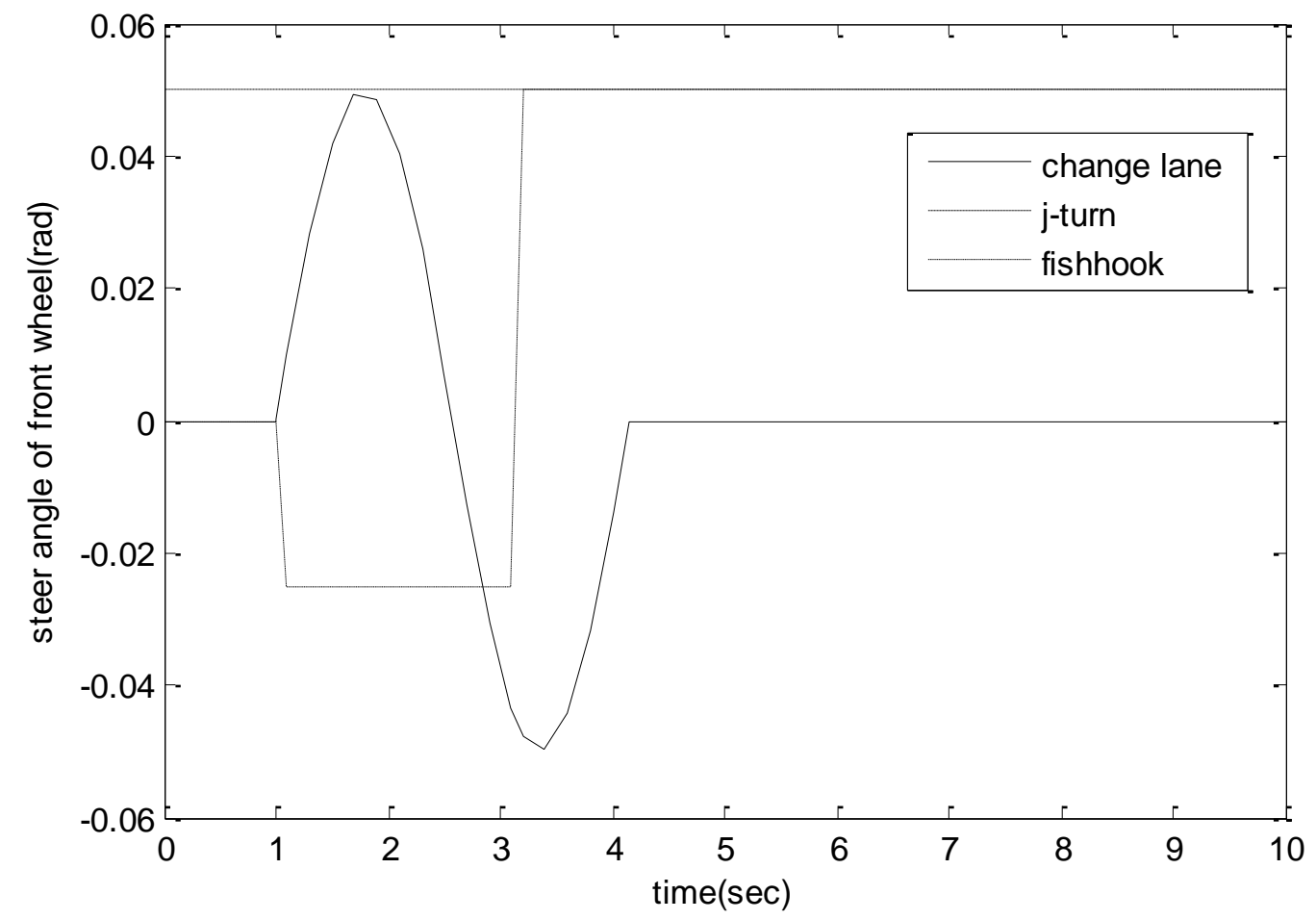

Figure 3. The evolution of the steering angle of the front wheel in J-turn, lane change and fish-hook steering manoeuvres.

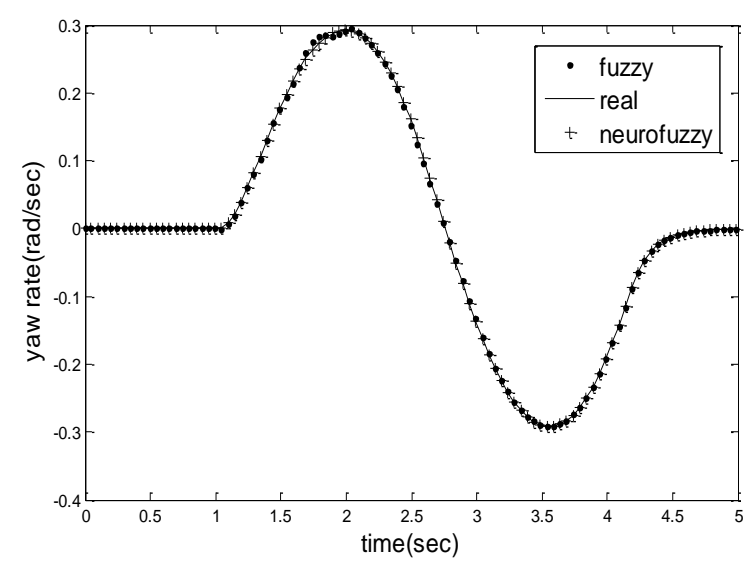

(a)

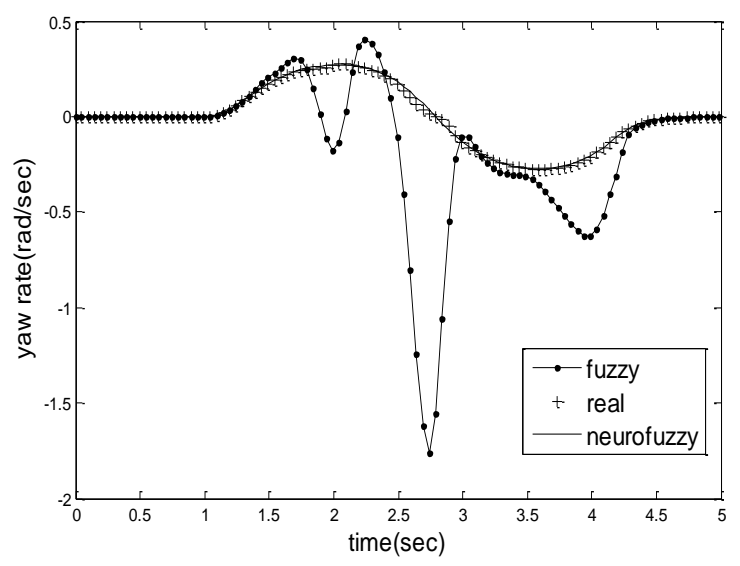

(b)

Figure 4. The yaw rate of the real (desired), fuzzy and neuro-fuzzy of the vehicle for the lane change manoeuvre on (a) dry and; (b) slippery road. 


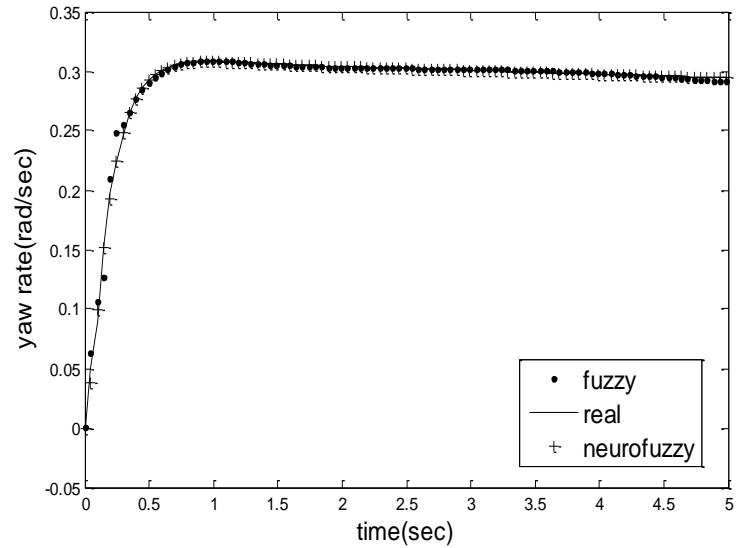

(a)

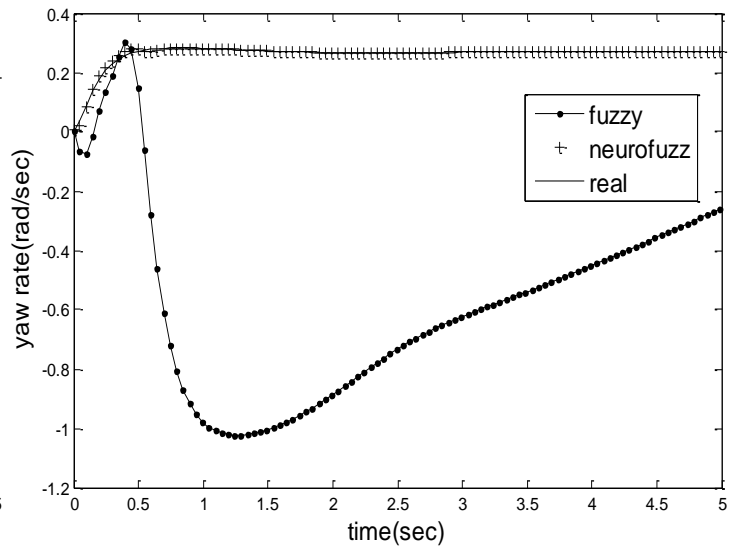

(b)

Figure 5. The yaw rate of the real (desired), fuzzy and neuro-fuzzy of the vehicle for the J-turn manoeuvre on (a) dry and (b) slippery road.

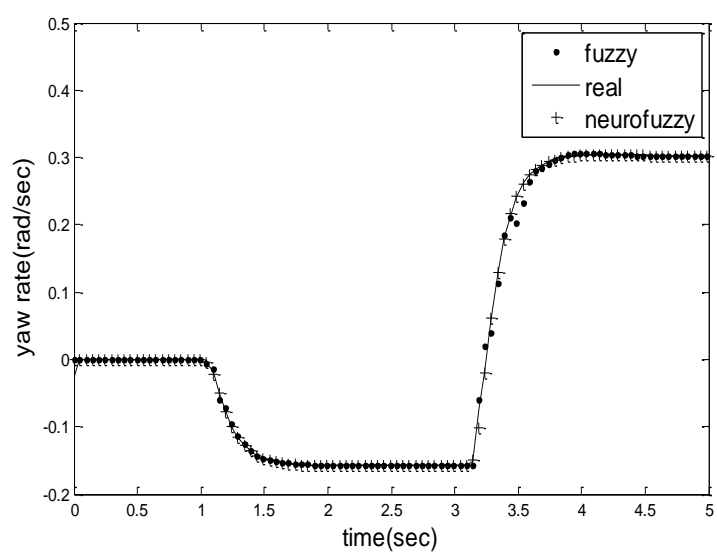

(a)

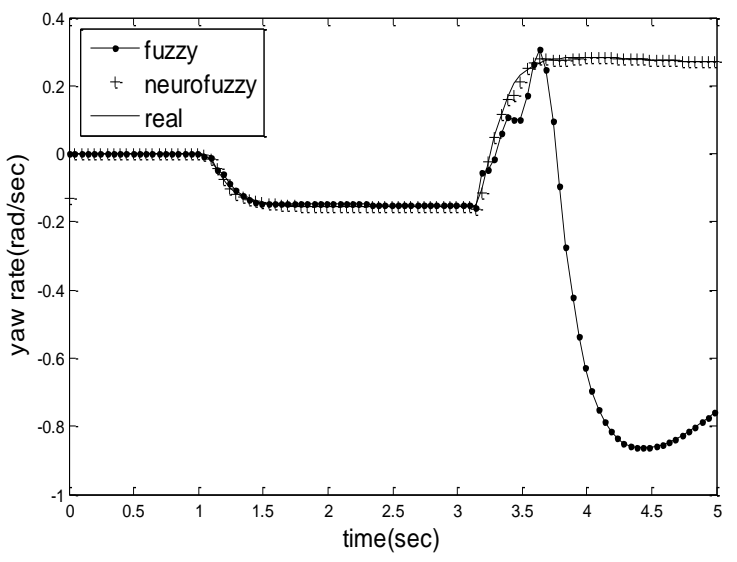

(b)

Figure 6. The yaw rate of the real (desired), fuzzy and neuro-fuzzy of the vehicle for the fish-hook manoeuvre on (a) dry and (b) slippery road.

Regarding the mentioned figures, the ability of the neuro-fuzzy algorithm to identify the uncertainties is quite clear relative to the case in which the fuzzy algorithm has been used alone. On dry roads, under regular conditions, there are no significant differences between the responses, but under uncertain conditions, the neuro-fuzzy identification algorithm can significantly steer the vehicles while the fuzzy one cannot. A precise look at Figure 4 to 6 , the slippery roads indicates that the fuzzy identifier is much worse at higher yaw rates of the vehicle or when there is a fast yaw rate change. These two reasons specify the role of the augmented neural network to enable the whole algorithm to have an acceptable and even accurate adaptation.

A quantitative analogy is also made between the results to help readers compare the algorithms better and more tangible (see Table 2). This table indicates how the integral of least squared error during a manoeuvre can grow and become large for untrained algorithm, i.e. the fuzzy one. In normal conditions, both algorithms present acceptable performance while in slippery roads, only the neuro-fuzzy identification algorithm is reliable and can be employed. Briefly, the results indicate the need for using an adaptation algorithm and process alongside fuzzy-based identification algorithms, especially for 
uncertain conditions. Here and in this article, we have used artificial neural networks for adaptation, but there is no restriction to use them.

Table 2. A Comparison between the performance of neuro-fuzzy and Fuzzy identification algorithms.

\begin{tabular}{|c|c|c|c|}
\hline Manoeuver/ model & Neurofuzzy & & Fuzzy \\
\hline \multicolumn{4}{|c|}{ Coefficient of friction $=1$} \\
\hline & \multicolumn{3}{|c|}{ Integral of least squared error during the manoeuvre } \\
\hline Change lane & $3.28 \mathrm{e}(-7)$ & & $2.35 \mathrm{e}(-5)$ \\
\hline J-turn & $7.19(-6)$ & & $1.44 \mathrm{e}(-4)$ \\
\hline fishhook & $1.70 \mathrm{e}(-5)$ & & $2.36 \mathrm{e}(-4)$ \\
\hline \multicolumn{4}{|c|}{ Coefficient of friction $=0.4$} \\
\hline \multirow[t]{2}{*}{ Manoeuver/ model } & Neurofuzzy & & Fuzzy \\
\hline & $\begin{array}{r}\text { Integral of least } \\
\text { the } m\end{array}$ & rror during & $\begin{array}{l}\text { Maximum of error (\%) } \\
\text { w.s.t. desired condition }\end{array}$ \\
\hline Change lane & $1.05 \mathrm{e}(-7)$ & 48.95 & 650 \\
\hline J-turn & $4.58 \mathrm{e}(-6)$ & 583.5 & 433 \\
\hline Fishhook & $8.01 \mathrm{e}(-6)$ & 371 & 383 \\
\hline
\end{tabular}

\section{CONCLUSION}

In this paper, a neuro-fuzzy identification algorithm was proposed to identify the handling of vehicles in uncertain road conditions. A model of car in CARSIM software was used as an almost real un-simplified model of a car. A set of steering data in regular condition together with some proper fuzzy rules and an artificial neural network were used to identify and train the identification algorithm to act as an efficient system at uncertain conditions. To show the effectiveness of the proposed algorithm, a comparison between the response of the system in the presence and absence of augmented neural network was made. The results showed the correctness and accuracy of the proposed neuro-fuzzy identification algorithm in three classical manoeuvres, i.e. lane change, J-turn, and fishhook at both certain and uncertain condition of roads within an acceptable error. In the case of using a fuzzy algorithm alone, desired performances were achieved for normal conditions only. At uncertain conditions, the fuzzy algorithm failed especially at higher yaw rate of the vehicle or when there was a fast yaw rate change. When the fuzzy algorithm is employed alone, the precision depends on how far we discretised our input and output, and foremost the precision of the model that consider each condition but in the case of using neurofuzzy one, the precision is almost guaranteed. Finally, the results pointed to the need for using an adaptation algorithm and process alongside fuzzy-based identification algorithms, especially for uncertain conditions.

\section{REFERENCES}

[1] Tseng H, Madau D, Ashrafi B, Brown T, Recker D. Technical challenges in the development of vehicle stability control system. Control Applications, 1999 Proceedings of the 1999 IEEE International Conference on: IEEE; 1999. p. 16606.

[2] Rajamani R. Vehicle dynamics and control: Springer Science \& Business Media; 2011. 
[3] Ackermann J, Bünte T, Odenthal D. Advantages of active steering for vehicle dynamics control. 1999.

[4] Kazemi R, Bahaghighat MK, Panahi K. Yaw moment control of four wheel steering vehicle by fuzzy approach. Industrial Technology, 2008 ICIT 2008 IEEE International Conference on: IEEE; 2008. p. 1-7.

[5] El Messoussi W, Pagès O, El Hajjaji A. Four-wheel steering vehicle control using takagi-sugeno fuzzy models. Fuzzy Systems Conference, 2007 FUZZ-IEEE 2007 IEEE International: IEEE; 2007. p. 1-6.

[6] Ma J, Liu G, Wang J. An AFS control based on fuzzy logic for vehicle yaw stability. Computer Application and System Modeling (ICCASM), 2010 International Conference on: IEEE; 2010. p. V5-503-V5-6.

[7] Yihu W, Dandan S, Zhixiang H, Xiang Y. A fuzzy control method to improve vehicle yaw stability based on integrated yaw moment control and active front steering. Mechatronics and Automation, 2007 ICMA 2007 International Conference on: IEEE; 2007. p. 1508-12.

[8] Canale M, Fagiano L, Milanese M, Borodani P. Robust vehicle yaw control using an active differential and IMC techniques. Control Engineering Practice. 2007; 15:923-41.

[9] Wang W, Zhao Y, Xu J, Liu W. Vehicle Handling Inverse Dynamics Model Identification Based on Neural Networks. Journal of Basic Science and Engineering. 2014;3:019.

[10] Zhang H, Wang J. Vehicle lateral dynamics control through AFS/DYC and robust gain-scheduling approach. IEEE Transactions on Vehicular Technology. 2016;65:489-94.

[11] Aalizadeh B, Asnafi A. Combination of particle swarm optimization algorithm and artificial neural network to propose an efficient controller for vehicle handling in uncertain road conditions. Neural Computing and Applications. 2018;30:58593.

[12] Aalizadeh B, Asnafi A. Integrated bees algorithm and artificial neural network to propose an efficient controller for active front steering control of vehicles. International Journal of Automotive \& Mechanical Engineering. 2016;13.

[13] Aalizadeh B. A neurofuzzy controller for active front steering system of vehicle under road friction uncertainties. Transactions of the Institute of Measurement and Control. 2018:0142331218780220.

[14] McAllister MN. Fuzzy logic with engineering applications (Timothy Ross). SIAM Review. 1996;38:174-5.

[15] Mamdani EH. Application of fuzzy algorithms for control of simple dynamic plant. Proceedings of the institution of electrical engineers: IET; 1974. p. 15858.

[16] Tanaka K, Wang HO. Fuzzy control systems design and analysis: a linear matrix inequality approach: John Wiley \& Sons; 2004.

[17] Lin C-T. A neural fuzzy control system with structure and parameter learning. Fuzzy Sets and Systems. 1995;70:183-212.

[18] Fullér R. Neural fuzzy systems. 1995.

[19] McCulloch WS, Pitts W. A logical calculus of the ideas immanent in nervous activity. The bulletin of mathematical biophysics. 1943;5:115-33.

[20] Moscovitch M. The hippocampus as a" stupid," domain-specific module: Implications for theories of recent and remote memory, and of imagination. 
Canadian Journal of Experimental Psychology/Revue canadienne de psychologie expérimentale. 2008;62:62.

[21] Rojas R. Neural networks: a systematic introduction: Springer Science \& Business Media; 2013.

[22] Regression B. New Tools for Prediction and Analysis in the Behavioral Sciences. Werbos, PJ https://books google se/books. 1974.

[23] Rumelhart DE, Hinton GE, Williams RJ. Learning representations by backpropagating errors. nature. 1986;323:533.

[24] Kriesel D. A brief introduction on neural networks. 2007.

[25] Hagan MT, Demuth HB, Beale MH, De Jesús O. Neural network design: Pws Pub. Boston; 1996. 